\title{
Couche limite dans le sillage d'une poche de cavitation
}

\author{
Boundary layer development downstream of a cavitation cavity closure. \\ JEAN-YVES BILLARD \\ Ecole navale / IRENAV (EA 3634) \\ BCRM Brest, CC 600 \\ 29240 BREST Cedex 9
}

Tél : +33 (0)2 982340 35, Fax : +33 (0)2 9823 38 57, e-mail : jean-yves.billard@ecole-navale.fr

HENDA DJERIDI

Laboratoire de physique des Océans (UMR 6523 CNRS/IFREMER/IRD/UBO)

Université de Bretagne Occidentale, UFR Sciences

6 Avenue Le Gorgeu BP 809, 29285 Brest Cedex

Tél : +33 (0)2 980183 12, Fax : +33 (0)2 9801 64 38, e-mail : henda.djeridi@univ-brest.fr

\section{CHRISTOPHE SARRAF}

Arts et Métiers ParisTech / LEMFI

51 Boulevard de l'Hôpital, 75013 Paris

Tél : +33 (0)2 982340 35, Fax : 33 (0)1 442463 98, e-mail : christophe.sarraf@ensam.eu

\begin{abstract}
$L$
e travail présenté concerne l'étude expérimentale des modifications d'une couche limite sur un profil NACA symétrique d'épaisseur relative $15 \%$ à Reynolds $0,510^{6}$, en présence d'une cavité de vapeur stable se développant sur l'extrados du profil près du bord d'attaque, pour un angle d'incidence de $10^{\circ}$. Une comparaison systématique des grandeurs intégrales et des grandeurs moyennes a été réalisée avec et sans poche de cavitation afin de proposer des mécanismes d'interaction entre la phase dispersée et la phase liquide. Les résultats obtenus montrent que la présence de la poche contribue à un épaississement de la couche limite et à une augmentation de la vitesse en très proche paroi. On observe un effet prépondérant du gradient de pression adverse et l'on confirme que la présence de la poche, en favorisant les échanges entre la zone de sillage et la zone de proche paroi, a un effet stabilisant sur l'écoulement.
\end{abstract}

$T$ The results of an experimental investigation of the turbulent boundary layer that develops on a symmetric hydrofoil at moderate Reynolds number when a stable partial cavity takes place in the vicinity of the leading edge at angle of attack of $10^{\circ}$, are presented. The modifications of the mean and turbulent properties introduced by the existence of the vapour phase have been compared to the same quantities in the non cavitating state. A particular focus has been put on the evolution of the logarithmic law of the velocity profile and on the modification of the global parameters The higher order moments have been studied to highlight the exchanges and transfers of turbulent quantities between the different regions of the boundary layer. The results have shown that the vapour phase modify the thickness of this zone of velocity defects and enhance the exchanges with the external layer leading to an increase of the velocity close to the wall. This particular phenomenon is related with a stabilisation of the boundary layer and delays its separation.

\section{I INTRODUCTION}

L'étude de la cavitation partielle sur profil bidimensionnel (poches cavitantes stables ou instables dont les dimensions restent inférieures à la corde du profil) a donné lieu à de très nombreuses publications. Une bibliographie importante sur ce sujet peut, en particulier être trouvée dans le travail col- lectif dirigé par Franc [1]. Les caractéristiques géométriques des poches ont été également largement étudiées notamment par Katz, [2] et Kawanami et al., [3]. La transition entre les comportements stable et instable des poches a été étudiée numériquement et expérimentalement $[4,5]$. De nombreuses données disponibles concernent les conditions d'écoulement dans la zone de fermeture proprement dite et quelques tra- 
vaux descriptifs portent sur la nature de la couche limite dans la zone d'ouverture de poche [6]. Les mécanismes conduisant à l'apparition de la poche revêtent un très grand intérêt ; Arakeri et Acosta [7] montrent que la poche en forme de bande doit sa stabilité à un bulbe de décollement laminaire. Cependant, bien que Zhang et al. [8] ou Guennoun [9] montrent que des germes siégeant dans des irrégularités de surface peuvent promouvoir l'apparition de bandes de cavitation dont la frontière amont est irrégulière, la question de l'apparition de poche en l'absence de bulbe n'est pas totalement fermée $[10,11,12]$. De plus, même si Dupont [13] observe que sur un profil particulier (NACA0009 modifié) une poche de vapeur accrochée a pour effet d'augmenter significativement la portance et que Kjeldsen et al.[14] observent un effet équivalent, peu de données expérimentales sont disponibles en ce qui concerne l'influence de la cavitation sur l'écoulement. En particulier très rares sont les travaux expérimentaux qui se sont intéressés à l'effet de la poche sur l'écoulement dans la zone située entre la fermeture de la poche et le bord de fuite.

Le présent travail s'attache à l'étude expérimentale des modifications d'une couche limite sur un profil NACA symétrique d'épaisseur relative $15 \%$ à Reynolds $0,510^{6}$, en présence d'une cavité de vapeur stable se développant sur l'extrados près du bord d'attaque lorsque le profil a une incidence de $10^{\circ}$. Les mesures du champ de vitesse en couche limite ont été réalisées par LDV. Les vitesses tangentielles moyennes ainsi que les quantités turbulentes sont systématiquement comparées pour mettre en évidence les différentes modifications engendrées par la présence de la phase dispersée en amont et dans le sillage de celle-ci. Une attention particulière est portée sur les mécanismes d'échanges et de transferts des quantités turbulentes dans la couche limite.

\section{II — DISPOSITIF EXPÉRIMENTAL}

Les essais ont été réalisés dans la veine du tunnel hydrodynamique (THEN), de section carrée de $192 \mathrm{~mm}$ de côté, et d'une longueur de $1 \mathrm{~m}$. Les mesures sont réalisées sur un hydrofoil symétrique NACA0015 [15] de longueur de corde $\mathrm{c}=100 \mathrm{~mm}$. Ces mesures de vitesse ont été effectuées par LDV 2 composantes dans un écoulement ensemencé par des particules d'Iriodine ${ }^{\circledR}$ de taille micrométrique. Le taux d'acquisition maximum est de $4 \mathrm{kHz}$ pour un temps de mesure maximum de $60 \mathrm{~s}$, les erreurs sur les composantes moyennes et fluctuantes restent respectivement inférieures à $1 \%$ et $1,5 \%$. Le volume de mesure est un ellipsoïde de dimension $40 \times 40 \times 800 \mu \mathrm{m}$. Les erreurs de biais spatial dues à la taille du volume de mesure en proche paroi du profil NACA sont estimées et restent inférieures à l'erreur statistique excepté dans la sous couche visqueuse. Il a été également vérifié que les composantes moyennes et fluctuantes ne sont pas significativement modifiées par la présence de micro-bulles dans le sillage de la poche. La grille de mesure, présentée en figure $1 \mathrm{a}$ ), orientée dans la direction des normales locales à la paroi, est très raffinée en proche paroi (pas de $50 \mu \mathrm{m}$ sur $1 \mathrm{~mm}$ en paroi) sur les seize normales réalisées. La poche de cavitation, représentée figure 1 b), est attachée à $\mathrm{x} / \mathrm{c}=0,05 \pm 0,01$ et a une longueur moyenne de $0,2 \pm 0,02 \mathrm{x} / \mathrm{c}$. Dans ces conditions de développement de la poche attachée, le paramètre de Thoma, $\sigma$, défini par :

$$
\sigma=\frac{P_{\infty}-P_{v}}{\frac{1}{2} \rho V_{\infty}{ }^{2}}
$$

où $\mathrm{P}_{\infty}$ et $\mathrm{V}_{\infty}$ représentent respectivement les conditions de pression et de vitesse à l'infini, $\rho$ la masse volumique $d u$ fluide et $\mathrm{P}_{\mathrm{V}}$ la pression de vapeur à la température de l'écoulement, est égal à 2,35 , valeur maintenue constante pour l'ensemble des résultats d'essais reportés.

\section{CARACTÉRISATION DE LA COUCHE LIMITE}

\section{III.1 EFFET DE LA POCHE SUR LES GRANDEURS INTÉGRALES}

L'état de la couche limite sur le profil NACA0015 est caractérisé par les grandeurs intégrales $\delta_{1}$ et $\delta_{2}$ (épaisseurs

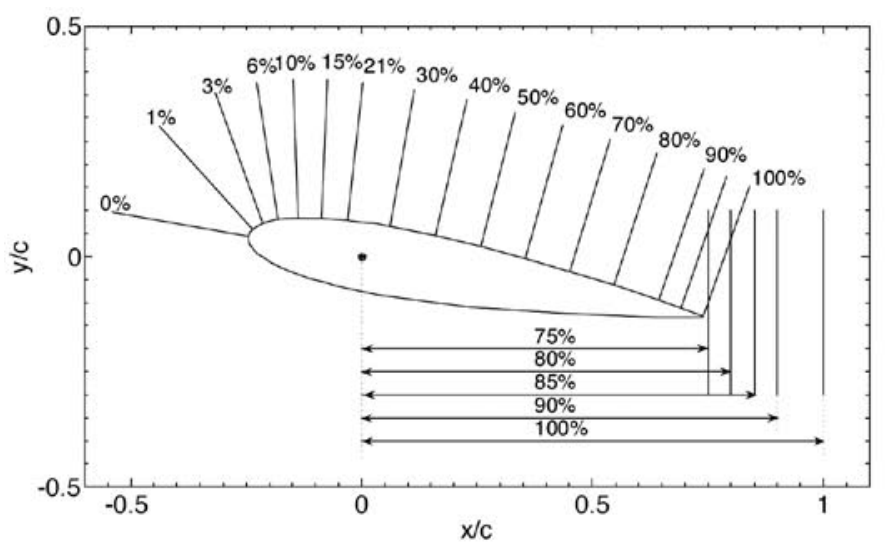

Figure 1 : a) Grille de mesure sur le profil et b) Conditions de cavitation.

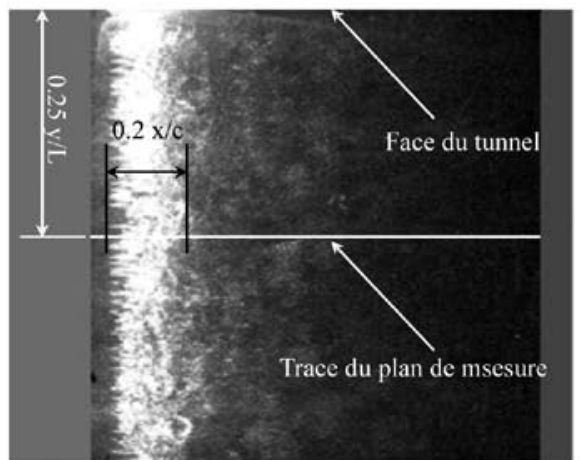


de déplacement et de quantité de mouvement définies par les équations (2)). L'épaisseur conventionnelle de couche limite et la vitesse tangentielle à la frontière extérieure sont respectivement notées $d$ et $\mathrm{U}_{\mathrm{e}}$, D'un point de vue pratique et compte tenu des conditions expérimentales dans une veine fermée et des incertitudes de mesure, $d$ et $\mathrm{U}_{\mathrm{e}}$ sont définis à partir de la vitesse maximale mesurée sur la normale à la paroi. Le paramètre adimensionnel $\mathrm{H}_{12}$, défini par les équations (2), est un paramètre caractéristique de la forme de la couche limite en fonction de son état laminaire ou turbulent [16].

$$
\delta_{1}=\int_{0}^{\delta}\left(1-\frac{\bar{u}}{U_{e}}\right) d y, \theta=\delta_{2}=\int_{0}^{\delta} \frac{\bar{u}}{U_{e}}\left(1-\frac{\bar{u}}{U_{e}}\right) d y, H_{12}=\frac{\delta_{1}}{\delta_{2}}
$$

Le facteur de forme a été déterminé avec et sans poche de cavitation et ses évolutions sont présentées en figure 2. L'évolution du facteur de forme en l'absence de poche montre que le plateau de $\mathrm{H}_{12}$ de la zone turbulente est légèrement supérieur à la valeur standard de 1,4 obtenue pour une couche limite turbulente. Le point de transition laminaire-turbulent est, dans ces conditions, localisé à $\mathrm{x} / \mathrm{c}=0,09$. En présence de la poche, on observe une modification du paramètre $\mathrm{H}_{12}$. En amont de la poche $\mathrm{H}_{12}$ franchit le seuil de décollement à 3,5 (régime laminaire) pour une position sur le profil $\mathrm{x} / \mathrm{c}=0,03$ et dans son sillage il prend une valeur voisine de 1,4. Par ailleurs le point de décollement de la couche limite turbulente, caractérisé par une valeur de $\mathrm{H}_{12}$ supérieure à 2,3, est repoussé vers le bord de fuite.

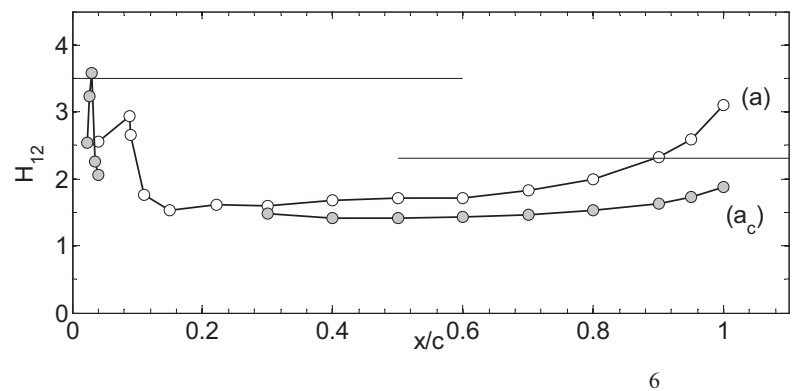

Figure 2 : Facteur de forme $\mathrm{H}_{12}, \mathrm{Re}=0,510^{6}$ et $\alpha=5^{\circ}$.

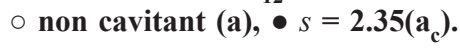

\section{III.2 DISTRIBUTION DE VITESSE DANS LA COUCHE LIMITE ET LOIS DE PAROI}

Pour une position donnée $\mathrm{x} / \mathrm{c}=0,8$ sur le profil, les distributions de la vitesse tangentielle dimensionnelle, présentées en figure 3, montrent que, en présence de la poche, la vitesse est accélérée en région de proche paroi dans un rapport maximum atteignant 1,25 à une distance à la paroi $\mathrm{y} / \mathrm{c}=0,005$. Dans le même temps la présence de la poche augmente l'épaisseur de la couche limite turbulente qui passe de 3 à $6 \mathrm{~mm}$ dans les conditions de la figure 3.

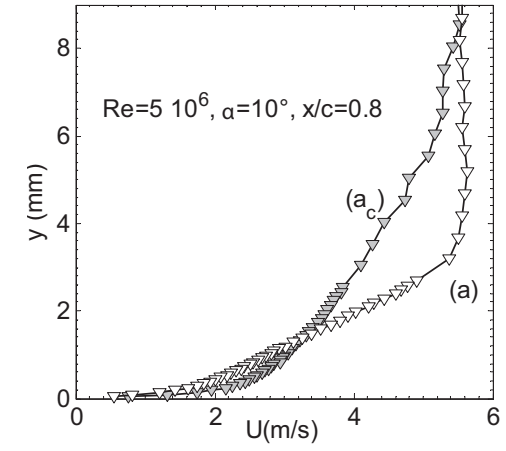

Figure 3 : Vitesses tangentielles dimensionnelles.

Les profils des vitesses moyennes tangentielles sont présentés sur la figure 4, en fonction des paramètres de paroi $\mathrm{U}^{+}$et $\mathrm{y}^{+}$. Ces paramètres adimensionnels sont définis de la façon suivante :

$$
U^{+}=\frac{\bar{u}}{U_{\tau}}, y^{+}=\frac{U_{\tau} y}{v}
$$

La valeur du coefficient de frottement est estimée par la corrélation semi empirique de Ludwieg-Tillmann [17] utilisant la valeur mesurée du paramètre $\mathrm{H}_{12}$ et un nombre de Reynolds calculé à partir de $\delta_{2}$ :

$$
C f=0.246 \cdot 10^{-0.678 H} \operatorname{Re}_{\delta_{2}}^{-0.268}, U_{\tau}=U_{e} \sqrt{\frac{C f}{2}}
$$

L'estimation de cette valeur étant essentielle les résultats de cette corrélation ont été comparés d'une part aux données expérimentales du gradient de vitesse à la paroi $\tau_{\omega}=\mu d U / d y$ et, d'autre part, aux résultats numériques obtenus par un calcul 3C3D [18] ; la convergence de ces trois résultats confirme que cette expression est bien adaptée à nos conditions expérimentales.

La distribution des vitesses tangentielles dans la zone interne d'écoulement turbulent est généralement désignée sous l'appellation de loi de paroi et obéit pour une couche limite de plaque plane sans gradient de pression à la relation :

$$
U^{+}=A \ln \left(y^{+}\right)+B
$$

Les données expérimentales sont reportées sur la figure 4 pour le cas non cavitant. L'ensemble des données permet d'obtenir une évolution linéaire de la vitesse conforme à la loi (5).

Cependant, en présence d'un gradient de pression adverse Mellor [19] a montré que le coefficient A, constant et égal à 5,75 dans le cas d'une couche limite turbulente sur plaque plane et sans gradient de pression, doit être modifié pour 


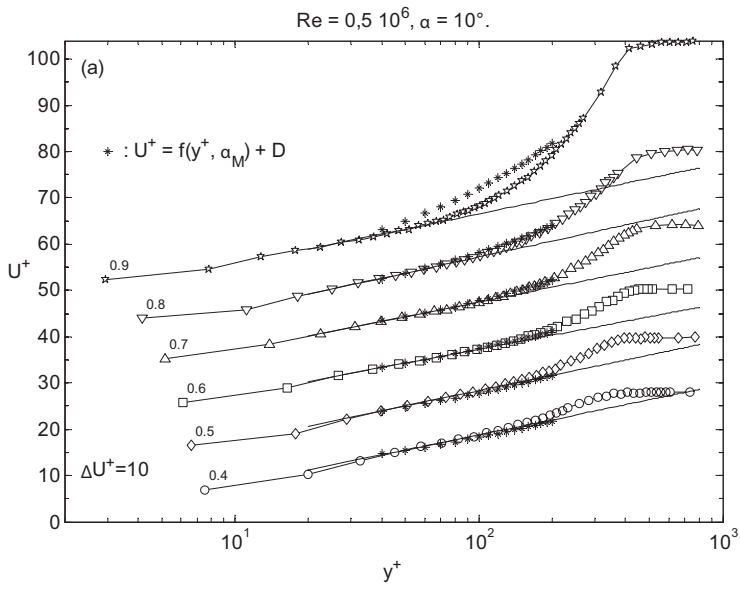

Figure 4 : Profils de vitesse en variable de paroi, comparaison à la loi de Mellor.

tenir compte de la présence du gradient de pression adverse. La loi modifiée s'écrit :

$U^{+}=u_{\mathrm{v}}^{+}+\frac{2}{\kappa}\left[\left(1+\alpha_{M} y^{+}\right)^{\frac{1}{2}}-1\right]+\frac{1}{\kappa} \ln \left[\frac{4}{\alpha_{M}} \frac{\left(1+\alpha_{M} y^{+}\right)^{\frac{1}{2}}-1}{\left(1+\alpha_{M} y^{+}\right)^{\frac{1}{2}}+1}\right]$

Dans cette expression $\alpha_{M}$ est un coefficient directement proportionnel au gradient de pression :

$$
\alpha_{M}=\frac{v}{\rho U_{\tau}^{3}} \frac{d p}{d x}
$$

Les données issues de nos essais ont été comparées avec succès à cette loi. Les valeurs des coefficients sont répertoriées dans le tableau 1.

Tableau 1 : Evolution de la valeur de la pente dans la zone logarithmique.

\begin{tabular}{|c|c|}
\hline Position $\mathbf{x} / \mathbf{c}$ & Valeur du coefficient $\mathbf{A}$ \\
\hline 0,5 & 10,3 \\
\hline 0,6 & 9,7 \\
\hline 0,7 & 10,8 \\
\hline 0,8 & 10,5 \\
\hline 0,9 & 8,7 \\
\hline 0,95 & 8,4 \\
\hline 1 & 8,9 \\
\hline
\end{tabular}

En présence de la poche, figure 5, on peut observer pour chaque position $\mathrm{x} / \mathrm{c}$ une distribution de vitesse en auto-simi- larité associée à une pente plus proche de la valeur A classiquement obtenue. Il semble que la présence de la poche tende ainsi à minimiser l'effet du gradient de pression adverse. On observe également sur cette figure, une diminution de l'amplitude de la zone de sillage et une extension de la zone logarithmique vers des distances à la paroi plus importantes. En effet, cette extension globale de la couche atteint $\mathrm{y}^{+}=1000$ en régime cavitant pour $\mathrm{y}^{+}=400$ sans la poche de cavitation. Cet effet correspond à un épaississement de la couche limite déjà mentionné.

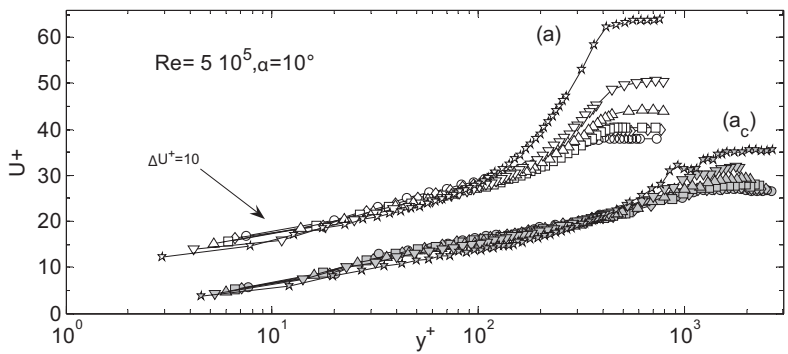

Figure 5 : Profils de vitesse en variable de paroi, régime non cavitant (a) et cavitant (ac).

\section{III.3 EFFET SUR LE GRADIENT DE PRESSION ET L'INTENSITÉ DU SILLAGE}

Le paramètre de pression de Clauser [20] prend en compte la forme de la couche limite et la valeur locale du coefficient de frottement :

$$
G=\frac{H_{12}-1}{H_{12} \gamma}, \gamma=U_{e} \sqrt{\frac{C_{f}}{2}}
$$

East et Sawyer ont proposé une caractérisation différente du gradient de pression à l'aide du facteur $\beta_{E . S .}[21]$. La forme de la couche limite, le coefficient de frottement local mais aussi le gradient de la vitesse extérieure interviennent explicitement dans cette expression :

$$
\beta_{E . S .} \equiv-\frac{2 \delta_{1}}{C f \cdot U e} \frac{d U e}{d x}
$$

Enfin le sillage peut être caractérisé par un paramètre proposé par Coles où $\Delta \mathrm{U}^{+}$représente l'amplitude de l'augmentation de vitesse par rapport à la loi log à l'extérieur de la couche limite :

$$
\Pi=\frac{1}{2} \kappa \Delta U^{+}
$$

Sur la figure 6 sont représentées les corrélations entre les paramètres d'East et Sawyer et de Coles d'une part (a) et entre les paramètres de Coles et Clauser d'autre part (b). La 

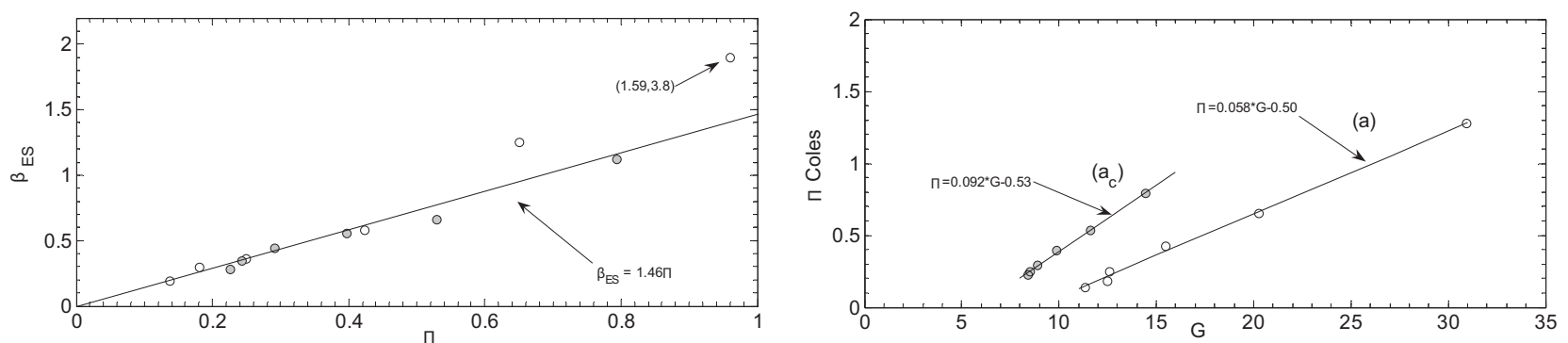

Figure 6 : effet de la poche sur les paramètres b, P, G

coïncidence des deux courbes sur cette première corrélation montre que la cavitation ne modifie pas la relation entre l'accélération dans la zone de sillage et le gradient de vitesse adverse. Cette absence d'influence peut s'expliquer par le fait que l'allure de la zone de sillage est directement liée, d'après Coles, au gradient de pression adverse. Par contre la forme de la couche limite est fortement modifiée par la présence de la poche, ce qui conduit sur la seconde corrélation à deux droites de pentes différentes. La forme de la couche limite a été affectée par la présence de la poche conduisant à une couche limite dont les caractéristiques sont beaucoup plus proches d'une couche limite pleinement développée sur plaque plane sans gradient de pression.

La présence de la poche modifie à la fois le gradient de pression, le sillage et la forme de la couche limite dans son sillage. Le sillage étant lié au gradient de pression, l'effet de la poche sur les deux paramètres $\beta$ et $\Pi$ est identique alors que la forme de la couche limite est indépendante du gradient de pression. ceci se traduit par une relation linéaire entre $\beta$ et $\Pi$ non modifiée en présence de la poche alors que la relation $(\Pi, \mathrm{G})$ reste linéaire mais ne présente pas de similarité avec et sans poche.

\section{III.3.1 Comparaison des quantités turbulentes}

Sur la figure 7 l'évolution en $\mathrm{y}+$ de la première composante normale du tenseur de Reynolds a été représentée sous forme non dimensionnelle en la rapportant au produit $U_{e} u_{\tau}$ suivant en cela la normalisation proposée par DeGraff et Eaton [22] qui permet la mise en évidence des phénomènes à la fois en proche paroi et dans la zone de sillage.

On remarquera que pour nos données cette normalisation permet de mettre en évidence le regroupement des courbes à la fois en proche paroi et dans la zone externe. Dans le cas non cavitant les deux courbes les plus proches du bord de fuite $(\mathrm{x} / \mathrm{c}=0.8$ et 0.9$)$ mettent en évidence l'effet du gradient de pression adverse. Dans le cas cavitant on observe une augmentation globale de la valeur du paramètre et l'on retrouve l'effet d'épaississement de la couche limite. De plus l'augmentation de l'intensité turbulente estompe l'effet du gradient de pression et toutes les courbes collapsent dans cette représentation.

Les mêmes remarques peuvent être faites à propos de la figure 8 où sont représentées les tensions de Reynolds en fonction du paramètre $\mathrm{y}^{+}$.

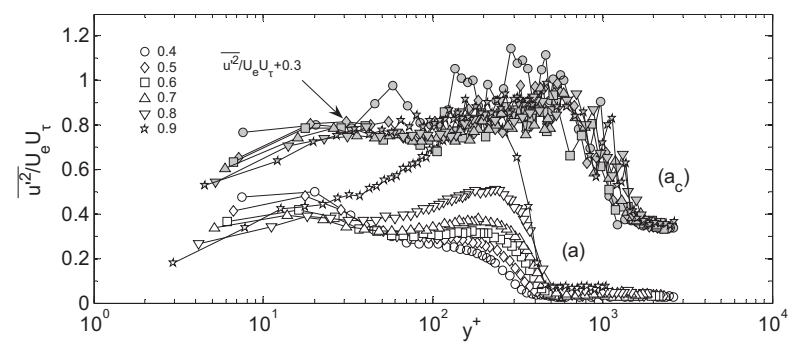

Figure 7 : Première composante du tenseur de Reynolds.

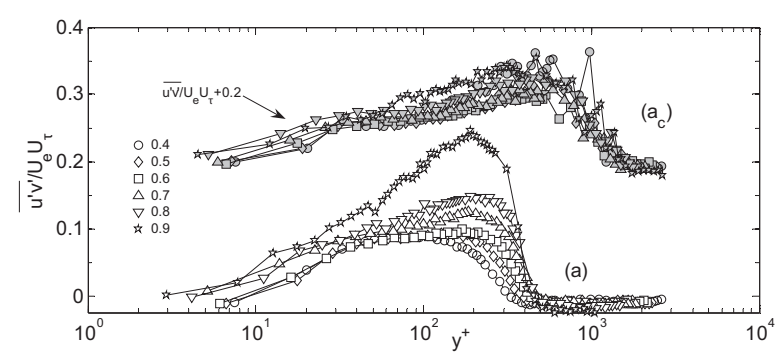

Figure 8 : Contrainte de cisaillement.

La figure 9 représente, dans les cas cavitant et non cavitant la corrélation des tensions de Reynolds. On y retrouve, dans le cas non cavitant un plateau à 0,4 dans la zone logarithmique, cette valeur est supérieure à la valeur habituellement obtenue $(0,3)$ dans le cas de la plaque plane. Dans le cas cavitant on obtient une valeur légèrement plus faible voisine de 0,3. L'effet de la poche se traduit donc par une

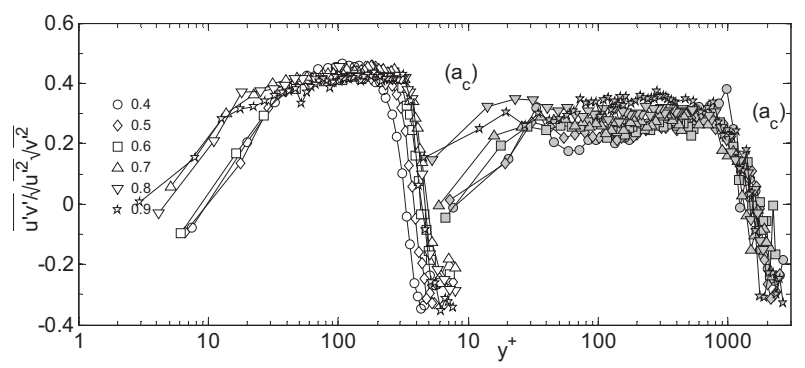

Figure 9 : Paramètre de corrélation des contraintes de Reynolds. 
diminution du paramètre qui reprend la valeur classiquement obtenue dans le cas de la plaque plane sans modifier la qualité de la corrélation sur l'ensemble de l'épaisseur de la couche limite.

Sur la figure 10 la production d'énergie cinétique turbulente a été reportée en fonction du paramètre $\mathrm{y}^{+}$. L'allure des courbes dans le cas non cavitant met en évidence une augmentation de la production dans la zone externe en particulier pour les normales les plus proches du bord de fuite. Dans le cas cavitant l'allure des courbes est beaucoup plus classique et l'on retrouve un comportement, dans la zone externe, pr0oche de la loi en $\left(\mathrm{Ky}^{+}\right)^{-1}$ obtenue sur plaque plane.

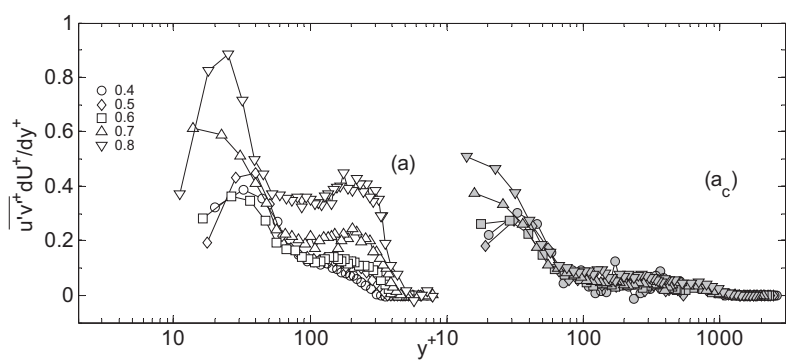

Figure 10 : Production d'énergie cinétique turbulente.

\section{CONCLUSIONS}

Les résultats du présent travail relatif à l'impact d'un développement de cavitation par poche au bord d'attaque d'un profil Naca0015 sur la couche limite dans son sillage ont montré que :

La présence de la poche conduit à un épaississement important de la couche limite et à une atténuation de l'effet du gradient de pression lié à la limitation différence de pression intrados - extrados ;

La poche ne modifie pas la zone de sillage qui s'adapte au nouveau gradient de pression qu'elle rencontre mais modifie la forme de la couche limite qui tend à se rapprocher d'une couche limite sur plaque plane. Là aussi on constate une atténuation de l'effet du gradient de pression adverse.

Concernant les quantités turbulentes, la présence de la poche tend à augmenter l'agitation turbulente dans la zone d'épaississement de la couche limite. Globalement, l'augmentation de la vitesse en paroi, la modification de la forme de la couche limite ainsi que la diminution du gradient de pression provoquée par la poche conduisent à une amélioration des mélanges dans la zone logarithmique, se traduisant par une amélioration de la stabilité de la couche limite et un retard au décollement.

\section{D RÉFÉRENCES}

[1] J.P. Franc, F. Avellan, B. Belahadj, J.Y. Billard, L. Briançon-Marjollet, D. Frechou, D.H. Fruman, A. Karimi, J.L. KuenY, J.M. Michel (1995 ) - "La cavitation mécanismes physiques et aspects industriels”. Presse Universitaire de Grenoble.

[2] J. KATZ (1984 ) — "Cavitation phenomena within regions of flow separation”. J. Fluid Mech 140 397-436

[3] Y. Kawanami , H. Kato , H. Yamaguchi , M. Maeda (1995 ) - "An experimental investigation of flow field around sheet cavity on foil section", Communication privée, Société Hydrotechnique de France, Section cavitation, LEGI, Grenoble, France.

[4] J.B. Leroux (2003 ) — "Etude expérimentale en tunnel hydrodynamique des instabilités de la cavitation par poche sur hydrofoil par la mesure spatio-temporelle du champ de pression pariétal", Thèse de doctorat, Ecole Centrale de Nantes - Université de Nantes, IRENav.

[5] R.E.A. Arndt, C.C.S. Song, M. KJeldsen, J. He, A. Keller (2000 ) — "Instability of partial cavitation : a numerical/experimental approach" . Proceedings of the 23 ${ }^{\text {rd }}$ Symposium on Naval Hydrodynamics, ONR, September, 17-22, Val de Reuil, France.

[6] J.P Franc , J.M. Michel (1985 ) — "Attached cavitation and the boundary layer - Experimental investigation and numerical treatment". J. Fluid Mech., vol 154 63-90

[7] V.H. Arakeri, A.J. Acosta (1973 ) — "Viscous Effects in the Inception of Cavitation on Axisymmetric Bodies" . Journal of Fluids Engineering 95 519-527

[8] Y. Zhang , S. Gopalan , J. Katz (1998 ) - "On the Flow Structure and Turbulence in the Closure Region of Attached Cavitation" . 22 ${ }^{\text {nd }}$ ONR Symp. on Naval Hydrodynamics

[9] M. F. GuENNOUN (2006 ) — "Etude physique de l'apparition et du développement de la cavitation sur un profil isolé". Thèse de l'Ecole Polytechnique Fédérale de Lausanne $n^{\circ} 3574$

[10] S. Gopalan , J. Katz (2000 ) — "Flow structure and modelling issues in the closure region of attached cavitation" Physics of Fluids, vol 12 (4 ) 896-911

[11] R.A. Furness, S.P Hutton (1975 ) — "Experimental and Theoretical Studies of Two-dimensional Fixed-type Cavities" . Journal of Fluids Engineering . $97 \quad 515-522$

[12] F. Avellan , P. Dupont , I. Rhyming (1998 ) — "Generation mechanism and dynamics of cavitation vortices downstream of a fixed leading edge cavity" . $17^{\text {th }}$ Symposium on Naval Hydrodynamics .

[13] P. Dupont (1991) _ “Etude de la dynamique d'une poche de cavitation partielle en vue de la prédiction de l'érosion dans les turbomachines hydrauliques", thèse de l'école polytechnique fédérale de Lausanne $n^{\circ} 931$.

[14] M. Kueldsen, R. E. A. Arndt, M. Effertz (2000)Spectral characteristics of sheet/cloud cavitation . Journal of Fluid Engineering . 122 481-487

[15] I. H. Aвbott , A.E. Von Doenhoff (1959) — "Theory of Wing Sections. Including a Summary of Airfoil Data”. Dover Publications, Inc .

[16] J. Cousteix (1989) - Turbulence et Couche limite, ed. Cepadues. 
[17] H. Ludwieg, W. Tillmann (1950 ) — "Investigation of the wall-shearing stress in turbulent boundary layer" . NACA Technical Memorandum . 1285

[18] R. Houdeville , P. Malecki (1994 ) - Calcul de couches limites tridimensionnelles. Description et mode d'emploi $d u$ code 3C3D, ONERA CERT, Septembre 1994.

[19] G.L. MELlor (1966) — “The effect of pressure gradients on turbulent flow near a smooth wall" . J. of Fluid Mech . 24(2) 255-274
[20] F. H. Clauser (1954 ) — "Turbulent boundary layer in adverse pressure gradients". J. Aero. Sci . 91-108

[21] L.F. EAST, W.G. SAWYER (1979 ) — "Turbulent boundary layers - Experiments, theory and modeling". $\mathrm{n}^{\circ}$. AGARD conference proceedings . 271 6-19

[22] D.B. DegraafF, J.K. Eaton (2000 ) — "Reynolds Number Scaling of the Flat-Plate Turbulent Boundary Layer" . J. Fluid Mech . 422 319-346 